\title{
Characterization of fracture properties of thin aluminum inclusions embedded in anisotropic laminate composites
}

\author{
Gabriella Bolzon \\ Department of Structural Engineering, Politecnico di Milano, Milano (Italy) \\ gabriella.bolron@polimi.it \\ Vladimir Buljak \\ Department of Strength of Materials, University of Belgrade - Faculty of Mechanical Engineering, Belgrade (Serbia)
}

Emanuele Zappa

Department of Mechanics, Politecnico di Milano, Milano (Italy)

\begin{abstract}
The fracture properties of thin aluminum inclusions embedded in anisotropic paperboard composites, of interest for food and beverage packaging industry, can be determined by performing tensile tests on non-conventional heterogeneous specimens. The region of interest of the investigated material samples is monitored all along the experiment by digital image correlation techniques, which allow to recover qualitative and quantitative information about the metal deformation and about the evolution of the damaging processes leading to the detachment of the inclusion from the surrounding laminate composite. The interpretation of the laboratory results is supported by the numerical simulation of the tests.
\end{abstract}

SOMMARIO. Le proprietà di frattura di inclusioni metalliche costituite da sottili stati di alluminio in compositi a base di carta, di interesse nel packaging alimentare, possono essere determinate mediante prove di trazione nonconvenzionali, condotte su campioni di materiale eterogeneo. La deformazione della regione di interesse nel corso della prova viene monitorata attraverso tecniche di correlazione d'immagini digitali che restituiscono informazioni quantitative, oltre che qualitative, sul fenomeno di progressivo distacco dell'inclusione metallica dal composito laminato che la circonda. L'interpretazione dei risultati sperimentali viene supportata dalla simulazione numerica delle prove di laboratorio.

KEYWORDS. Fracture properties; Aluminium; Laminates; Paperboard composites.

\section{INTRODUCTION}

$\mathrm{M}$ aterials widely exploited in food and beverage packaging industry consist of layered composites, made of paperboard foils coupled with aluminium and polymer plies. Paperboard production process consists of the lamination of a network of interconnected fibres, which acquire a preferential orientation in the lamination (machine) direction. Thus, the material presents anisotropic overall characteristics, which are transferred to the layered composite formed by further lamination. The final packaging material is also inhomogeneous, due to the different ply sequence provided, for instance, in correspondence of the cap opening areas of beverage packages. The mechanical properties of this complex material system must be adequate to sustain the stresses induced by folding, forming and filling industrial processes without apparent damaging. Non-conventional testing procedures have been therefore designed in 
order to characterize the mechanical properties and to follow the evolution of the damaging phenomena leading to failure of the composite and of its constituents. A brief overview of current research activities in this field is presented in [1]. This communication focuses on the procedures implemented for the determination of the fracture properties of the thin aluminium inclusions embedded in the anisotropic paperboard composites in the cap opening areas. The sought mechanical characteristics are deduced from the results of tensile tests performed on strips of the heterogeneous material under consideration. The main information about the deformation and the damaging phenomena developing in the specimens is collected by the continuous monitoring of the region of interest by a digital camera. Snapshots are processed by Digital Image Correlation (DIC) techniques, introduced in [2], to recover quantitative data.

DIC techniques permit to reconstruct the whole deformation history of the specimen in the field of view of the camera with high accuracy in contact-less mode. These interesting characteristics foster the continuous development of the methodology with the reduction of the elaboration time, the optimization of the computer algorithms and the improvement of the measurement precision. Consequently, the application field of this technique is progressively enlarged and recent contributions include metals [3], wood [4], transparent media [5] and plastics [6, 7] among others.

In this investigation, the interpretation of the large amount of experimental data recovered by DIC is supported by the numerical simulation of the tests. Fracture properties are then inferred though inverse analysis tools, as illustrated in next Sections.

\section{EXPERIMENTAL PROCEDURES}

he mechanical response of paper-based materials is usually recovered from tensile tests, performed according to Standards [8] on strips of dimensions $15 \times 180 \mathrm{~mm}^{2}$ cut from the material batches to be investigated. This experimental setup has been exploited also for the characterization of the aluminium laminate inclusion shown in Fig. 1(a) in a configuration close to ultimate failure. The material under investigation consists of a thin aluminium foil (a few $\mu \mathrm{m}$ thick) coated by polymeric layers and embedded in the paperboard composite. The main mechanical degradation phenomenon of this material system consists of the separation of the aluminium foil in the laminate inclusion at the interface with the paperboard composite.

The standard tensile test is carried out under displacement control. The equipment returns the load-displacement curve shown in Fig. 2, which reflects the overall behaviour of the heterogeneous specimen all along the experiment. In particular, the long plateau on the tail of the curve represents the residual load carrying capacity of the specimen due to the presence of the polymeric layers, which undergo large deformations after their separation from the stiffer aluminium foil.

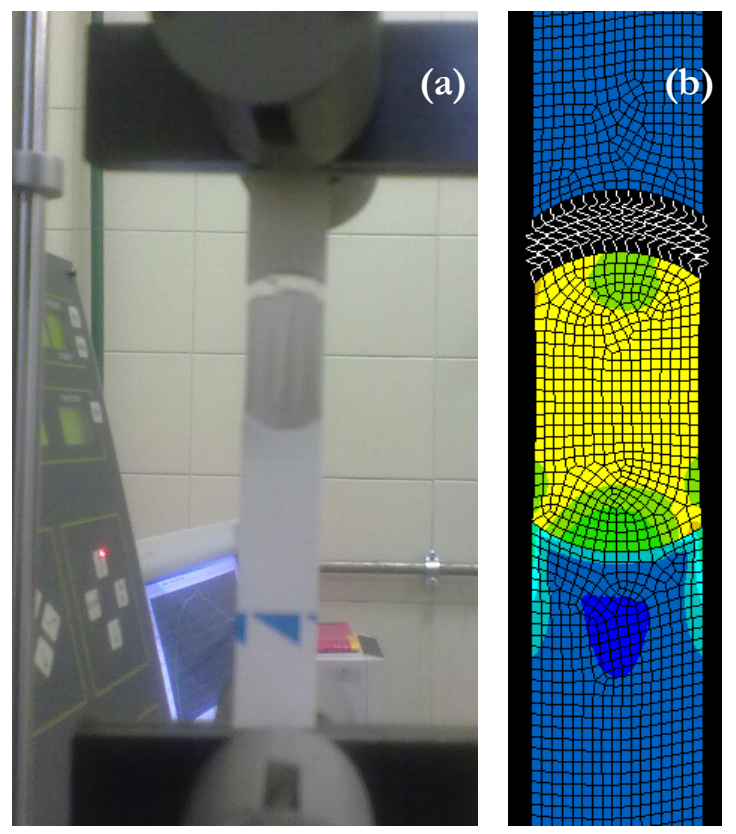

Figure 1: Failure of a heterogeneous specimen under tensile test (a) and the finite element simulation of the experiment (b). 


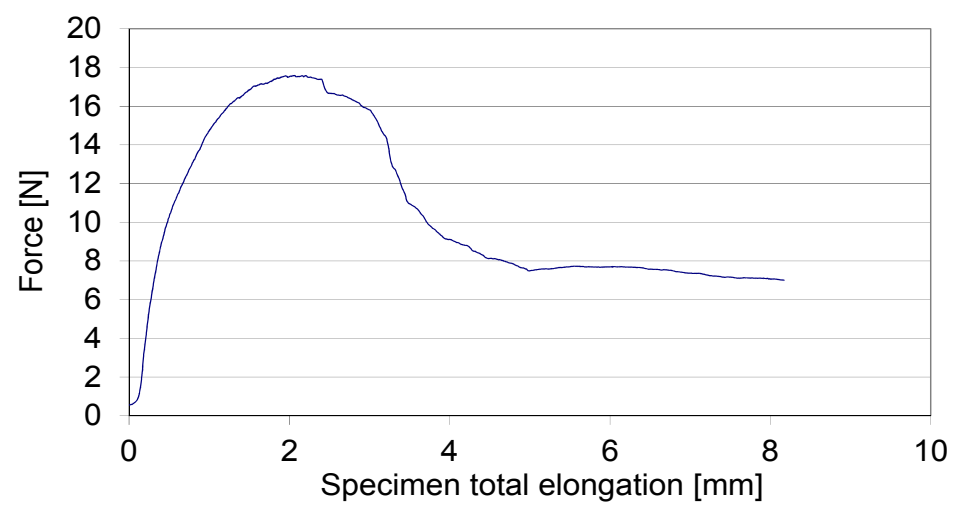

Figure 2: Force versus total specimen elongation in tensile test.

The region of interest in the investigated material sample is monitored by a digital camera (Allied GX3300, with a $3296 \times$ 2472 pixel 4/3" CCD sensor) equipped with $50 \mathrm{~mm}$ Zeiss optics. The photographs are acquired with a grab frequency of $1 \mathrm{~Hz}$ for the full length of the test. A snapshot sequence is represented in Fig. 3. These images can be processed by Digital Image Correlation (DIC) techniques, as introduced in [2]. The reliability of the method is improved by a speckle pattern produced on the specimen surface [9], visible in Fig. 3.

DIC permits to recover the displacement field developing in the region of interest by the comparison of two subsequent images of the marked specimen, acquired in the course of the experiment at different loads. The first picture, assumed as a reference, is split into rectangular areas (subsets) of fixed size. The subset size can change substantially with the experimental conditions [10], commonly in the range between $11 \times 11$ and $45 \times 45$ pixel. In this application, the subset size is $24 \times 24$ pixel.

DIC algorithm is applied to each subset of the reference picture in order to find the second image portion which is the 'most similar' to the starting one, i.e. the region that corresponds to the minimum value of a proper figure of merit, which assumes small values for similar images and larger values in the opposite cases. A proven effective correlation algorithm [10] minimizes the quadratic difference between the intensity pattern of each subset of the first (reference) image and of the next (deformed) image portions. This approach was chosen for this work.
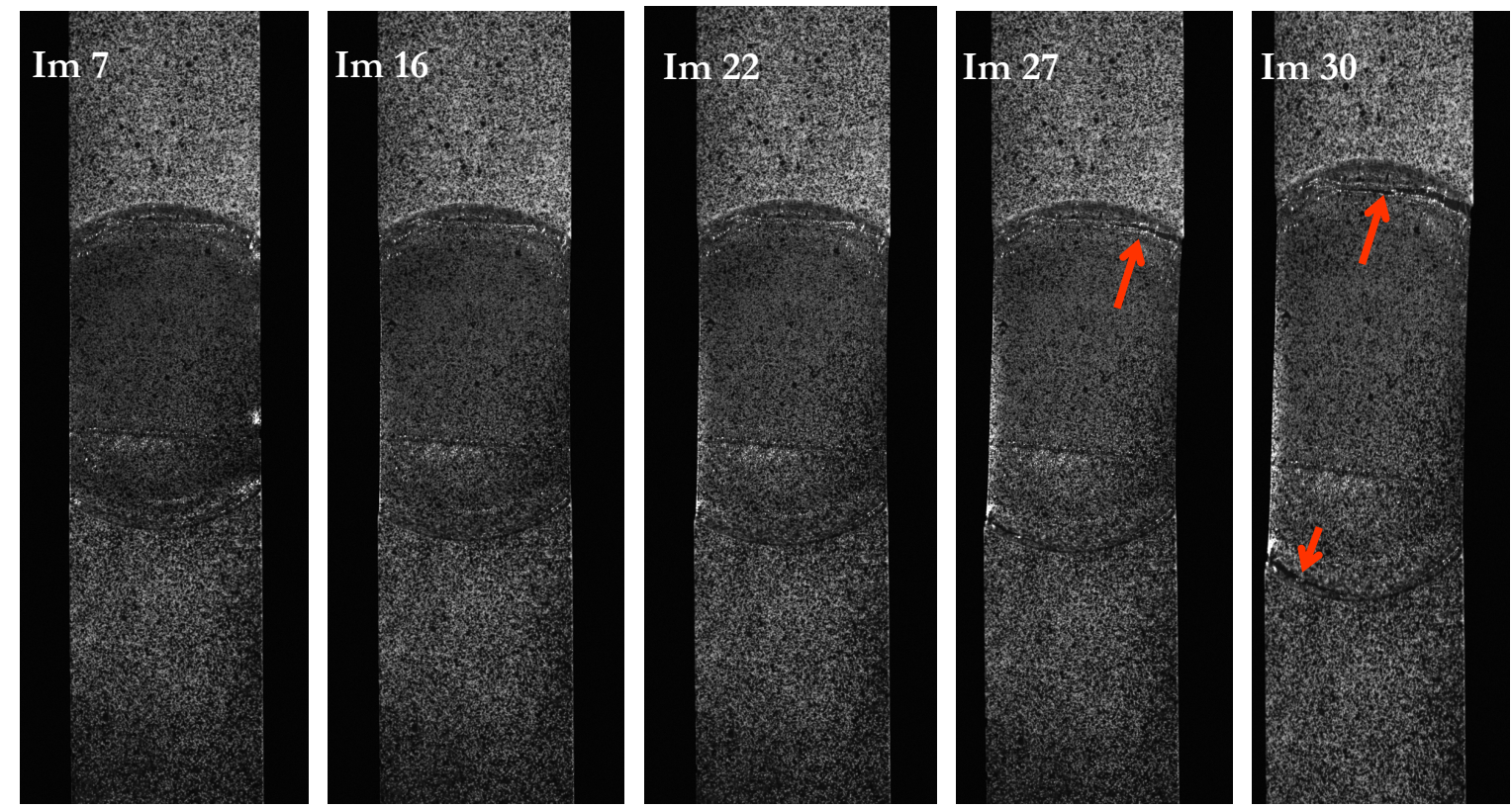

Figure 3: Sequence of digital images showing crack appearance and evolution during the test.

DIC techniques include the possibility to apply a deformation to the subset of the first image in order to better fit a portion of the second image and therefore improve the accuracy of the results. A further improvement results from the 
overlap of two contiguous subsets of the reference image, both in horizontal and in vertical direction. This overlap allows to increase the resolution while maintaining a relatively large subset size. In fact, too small subsets may even decrease the reliability of the results [11]. In this work, the overlapping region was assumed equal to $50 \%$ of the subset size in both directions.

An example of DIC results is shown in Fig. 4, which represents the displacement field in the heterogeneous specimen during the tensile test (according to the image orientation, the right strip end is fixed, while the displacement is imposed to the left end). Notice that, to improve readability, only one out of 16 sampled results are shown in the figure by arrows superimposed to the original specimen picture.

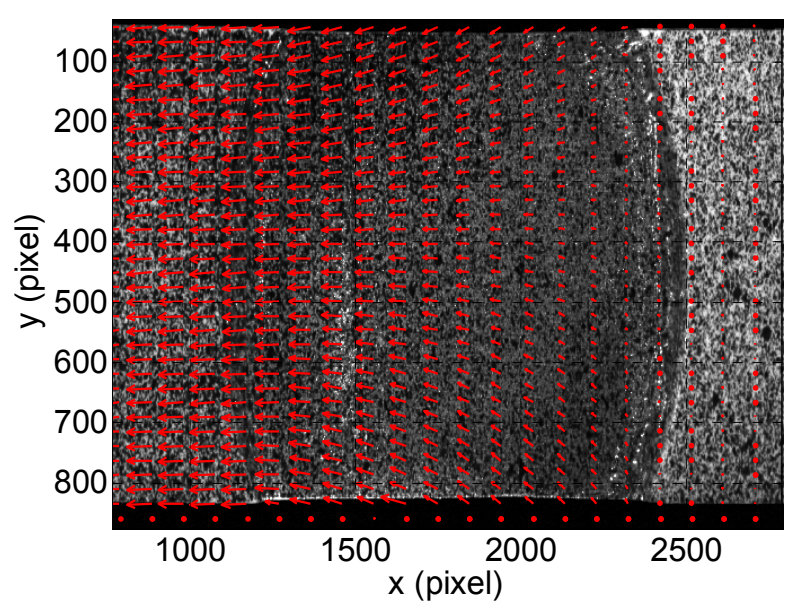

Figure 4: Detail of the displacement field obtained by DIC from the comparison of images 7 (the assumed reference one) and 16 (loaded specimen head on the left, fixed on the right); notice the different scales along the horizontal and the vertical direction.

The displacement field can be given the alternative representation of Fig. 5, where plots rely on all available data. The pictures suggest that most deformation concentrates in the area of the aluminium laminate inclusion, while the stiffer paperboard composite transfers the load undergoing an almost rigid body motion. The information provided in Fig. 4 and Fig. 5 also suggests that most part of the aluminium inclusion is almost uniformly stretched in the loading direction and experiences much smaller lateral contraction in the transversal direction, as expected.
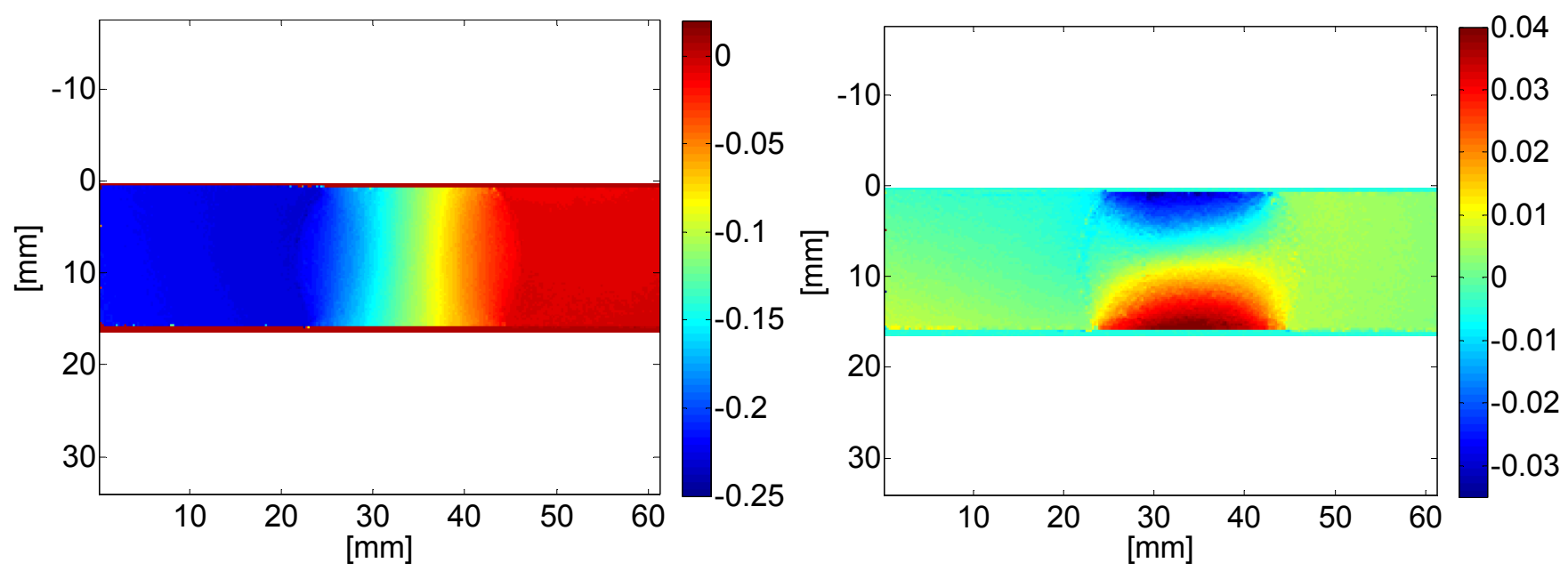

Figure 5: The displacement field in the heterogeneous specimen under tensile test (loaded specimen head on the left, fixed on the right), reconstructed by the correlation of the digital image 7 (the reference one) and 16 acquired during the test (see Fig. 3).

The situation evolves progressively as suggested for instance by Fig. 6, which visualises the displacement field in the aluminium inclusion reconstructed from the digital image 22, see Fig. 3. Even if not yet visible in the corresponding snapshot, DIC analysis evidences the onset of the degradation phenomena leading to the progressive separation of the 
aluminium foil and to the ultimate failure of the specimen. The appearance and evolution of the interfacial cracks are then clearly evidenced, for instance in the photographs 27 and 30 reported in Fig. 3.
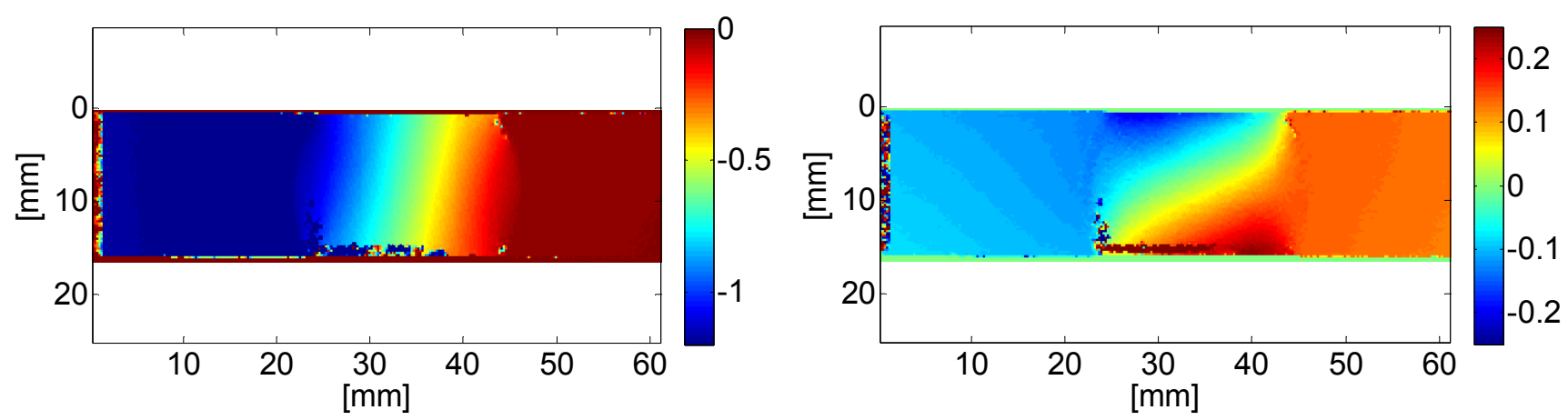

Figure 6: The displacement field in the heterogeneous specimen under tensile test (loaded head on the left, fixed on the right), reconstructed by the correlation of the digital images 7 (the reference one) and 22 acquired during the test (see Fig. 3).

DIC permits to follow the evolution of the displacement field all along the test in all locations of interest and to process this information for subsequent analyses. Opening and sliding relative displacements associated with the material separation process can be quantified by focusing attention on different material lines, for instance those sketched in Fig. 7. Results are represented in Figs. 8 and 9: the graphs in Fig. 8 show the evolution of the left (a) and of the right (b, c) boundary between the aluminium laminate and the paperboard composite during the tensile test, while the sequence of Fig. 9 evidence the relative displacements between the paperboard composite and the aluminium laminate, produced by removing the initial offset between the reference lines. This kind of information permits the identification of the fracture properties, inferred by the comparison with the result of the numerical simulation of the test.

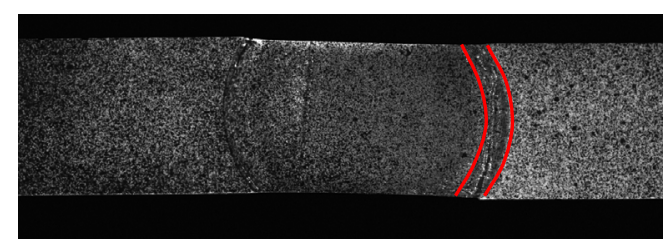

Figure 7: Reference lines for the recovery of the relative displacements during material separation.

\section{SIMULATION OF THE LABORATORY TESTS}

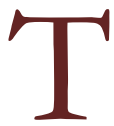
he interpretation of the experimental results can be supported by the numerical simulation of the test, performed e.g. by the Finite Element (FE) model visualised in Fig. 1(b). Properly calibrated elastic - plastic constitutive models can interpret the response of the isotropic aluminium laminate and of the orthotropic paperboard composite, while the progressive fracture process can be simply simulated by non - linear spring elements, endowed with the piecewise linear traction - separation law schematized in Fig. 10(a), according to a cohesive - crack approach earlier exploited in different fracture mechanics contexts $[12,13]$.

In this investigation, classical von Mises plasticity model with exponential hardening and saturation strength is assumed to describe the continuum behaviour of the investigated aluminium laminate. The corresponding constitutive properties are deduced from independent laboratory tests: $3150 \mathrm{MPa}$ equivalent elastic modulus; $5.45 \mathrm{MPa}$ initial yield limit; $17.95 \mathrm{MPa}$ ultimate strength; 34.5 hardening exponent.

The considered cohesive law is characterized by five independent parameters (see Fig. 10a), which represent: the maximum force $\mathrm{F}_{\max }$ transmitted across the interface between the aluminium laminate and the paperboard composite at $0.5 \mathrm{~mm}$ separation distance, a value that accounts for the material deformability between the reference lines assumed on the paper and aluminium sides; the intermediate force $\mathrm{F}_{1}$ that corresponds to the additional separation distance $\mathrm{D}_{1}$; the residual force $\mathrm{F}_{\text {plat }}$ carried by the polymer coating beyond further separation $\mathrm{D}_{2}$. 

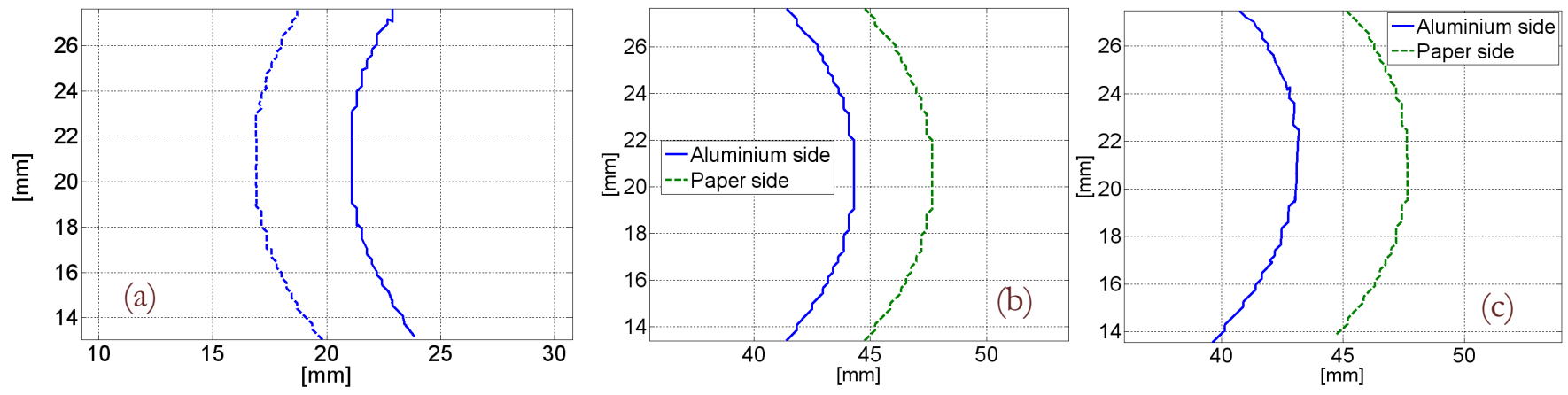

Figure 8: Evolution of the left (a, continuous line: reference image 7; a, dashed line: image 22 ) and of the right (b: reference image 7, c: image 40) boundary between the aluminium laminate and the paperboard composite during the tensile test; in (b) and (c), two reference lines close to the interface where fracture develops (one on the paper side, one on the aluminium side) are separately monitored.
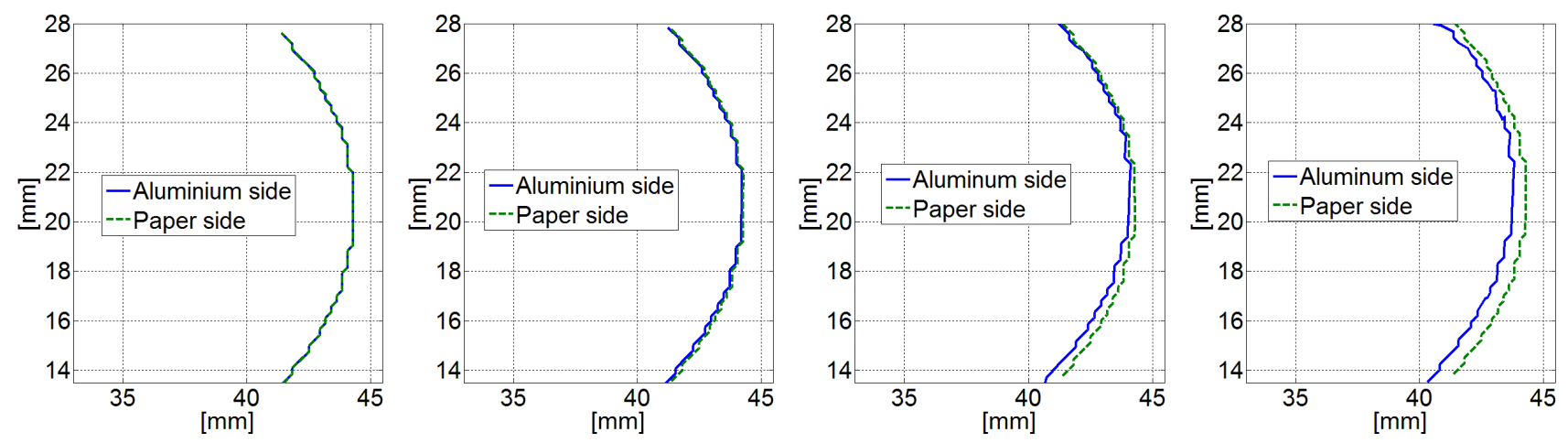

Figure 9: Recovery of the relative displacements during material separation from images 7, 22, 27, 32 (left to right).

The actual values of the fracture parameters relevant to the investigated material samples can be recovered by the inverse analysis procedure introduced in the next Section, based on the comparison between the results of the experiment and the corresponding output of the test simulation. The reduced FE model shown in Fig. 10(b) is implemented to this purpose.

The investigated domain consists of the aluminium inclusion only. Suitable boundary conditions, deduced from the performed DIC measurements (shown in Figs. 8 and 9), are applied along the lines (coloured in Fig. 10b) that represent the interface with the surrounding packaging material. In this way, the cumbersome calibration of the mechanical properties of the paperboard composite is avoided and the relevant uncertainties do not affect the identification result. The FE model is completed by the insertion of non-linear spring elements along the side where fracture propagates.
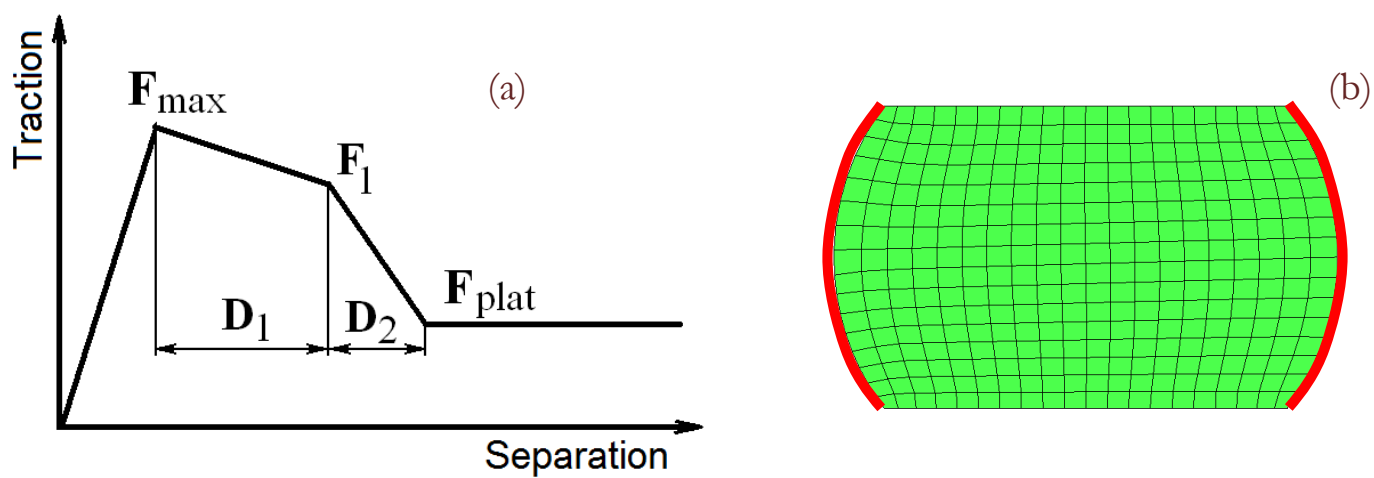

Figure 10: Schematic representation of the assumed traction - separation law and the reduced FE model exploited for identification purposes. 


\section{CALIBRATION OF THE FRACTURE PROPERTIES}

$\mathrm{P}$ arameters $\mathrm{F}_{\max }, \mathrm{F}_{1}, \mathrm{~F}_{\text {plat }}, \mathrm{D}_{1}$ and $\mathrm{D}_{2}$ (introduced above) characterize the response of the equi-distanced springs, which allow to simulate the fracture propagation in the aluminium laminate. Their calibration is based on the data collected during the tensile tests, namely: the load-displacement curve visualised in Fig. 2 and the progressive mismatch between paper and aluminium boundary, deduced from the analysis of the digital images and partly reported in the graph sequence of Fig. 9. Since snapshots are acquired at a fixed frequency and the loading velocity is also known, a correspondence can be established between each snapshot and the points along the overall force-displacement curve.

The sought parameters can be recovered by an inverse analysis procedure [14] based on the minimization of the quadratic discrepancy function $\omega$, which accounts for two contributions: $\omega_{C}$ relevant to the crack opening profiles deduced from snapshots $7-41$ (see e.g. Fig. 9); $\omega_{L}$ defined by force levels sampled over a grid of 100 equally spaced points along the displacement axis of the load - elongation curve (Fig. 2):

$$
\omega=\omega_{C}+\omega_{L}=\frac{1}{2}\left[\mathbf{d}_{C}^{T} \cdot \mathbf{d}_{C}+\mathbf{d}_{L}^{T} \cdot \mathbf{d}_{L}\right]
$$

In the above expression, $\mathbf{d}_{C}, \mathbf{d}_{L}$ represent dimensionless (normalized with respect the units) residual vectors, evaluated as the difference between physical quantities measured in the experiment and computed by the simulation, as a function of the sought parameters: $\mathbf{d}_{C}$ collects opening displacements originally evaluated in $\mathrm{mm}$ and $\mathbf{d}_{L}$ forces originally evaluated in $\mathrm{N}$.

The minimization of the discrepancy function $\omega$ is performed by a gradient - based algorithm, starting from different initialization vectors, summarized in Tab. 1, to single out the likely occurrence of local minimum points. The parameter values obtained at convergence are also listed in Tab. 1. These numerical results are conventionally rounded to the third decimal figure, regardless to the uncertainty of the estimation.

The most recurrent among the different solutions, graphically represented in Fig. 11 (left), correspond to almost equivalent local minimum points while the lowest (optimum) value $\omega_{\text {opt }}$ of the discrepancy function is recovered from the second initialization. However, notice that all solutions practically return the same estimation of the parameter $F_{\max }$, which reflects the overall material strength.

The graphs in Fig. 11 (right) compare the experimental output with the load - displacement curves reconstructed on the basis of the converged parameter values listed in Tab. 1. Notice that all computed material responses constitute a reasonable approximation of the real behaviour.

The informative content of the DIC measurements can be appreciated from the results listed in Tab. 2 and graphed in Fig. 12, which refer to identification results recovered by including the contribution only in the discrepancy function.

Notice that the parameter $F_{\max }$ is identified with the same accuracy as in Tab. 1 and that the experimental load elongation curve is closely reproduced in the only significant portion that corresponds to the exploited information range: in fact, the last considered digital image (41) corresponds to about $3 \mathrm{~mm}$ overall displacement.

\begin{tabular}{llcccccc}
\hline & & $\mathbf{F}_{\max }[\mathrm{N}]$ & $\mathbf{F}_{1}[\mathrm{~N}]$ & $\mathbf{F}_{\text {plat }}[\mathrm{N}]$ & $\mathbf{D}_{1}[\mathrm{~mm}]$ & $\mathbf{D}_{2}[\mathrm{~mm}]$ & $\begin{array}{c}\boldsymbol{\omega}_{\text {opt }} \\
\left(\omega_{C}, \omega_{L}\right)\end{array}$ \\
\multirow{2}{*}{ Trial 1 } & Initialization & 0.6 & 0.54 & 0.2 & 1.52 & 5 & $\mathbf{6 3 . 2 9}$ \\
& Converged & $\mathbf{1 . 1 2 9}$ & $\mathbf{1 . 0 5 4}$ & $\mathbf{0 . 4 2 7}$ & $\mathbf{0 . 8 4 7}$ & $\mathbf{3 . 5 1 0}$ & $(39.31+23.98)$ \\
\multirow{2}{*}{ Trial 2 } & Initialization & 0.75 & 0.483 & 0.45 & 1.6 & 9 & 48.64 \\
& Converged & $\mathbf{1 . 1 5 0}$ & $\mathbf{0 . 5 7 5}$ & $\mathbf{0 . 3 1 6}$ & $\mathbf{2 . 3 7 5}$ & $\mathbf{1 0 . 0 6 4}$ & $(32.19+16.45)$ \\
\multirow{2}{*}{ Trial 3 } & Initialization & 0.55 & 0.44 & 0.05 & 1.4 & 3.4 & $\mathbf{6 3 . 2 8}$ \\
& Converged & $\mathbf{1 . 1 2 6}$ & $\mathbf{1 . 0 4 6}$ & $\mathbf{0 . 4 2 7}$ & $\mathbf{0 . 8 9 4}$ & $\mathbf{3 . 5 0 4}$ & $(39.33+23.95)$ \\
\multirow{2}{*}{ Trial 4 } & Initialization & 0.85 & 0.1 & 0.02 & 1.1 & 5.5 & $\mathbf{6 5 . 9 5}$ \\
& Converged & $\mathbf{1 . 1 5 0}$ & $\mathbf{0 . 9 2 4}$ & $\mathbf{0 . 4 2 7}$ & $\mathbf{1 . 2 3 6}$ & $\mathbf{3 . 4 3 4}$ & $(41.60+24.35)$ \\
\hline
\end{tabular}

Table 1: Initialization vectors and parameter values returned by the minimization of the discrepancy function $\omega$ including contributions from both the crack opening profile $\left(\omega_{C}\right)$ and from the load - elongation curve $\left(\omega_{L}\right)$. 

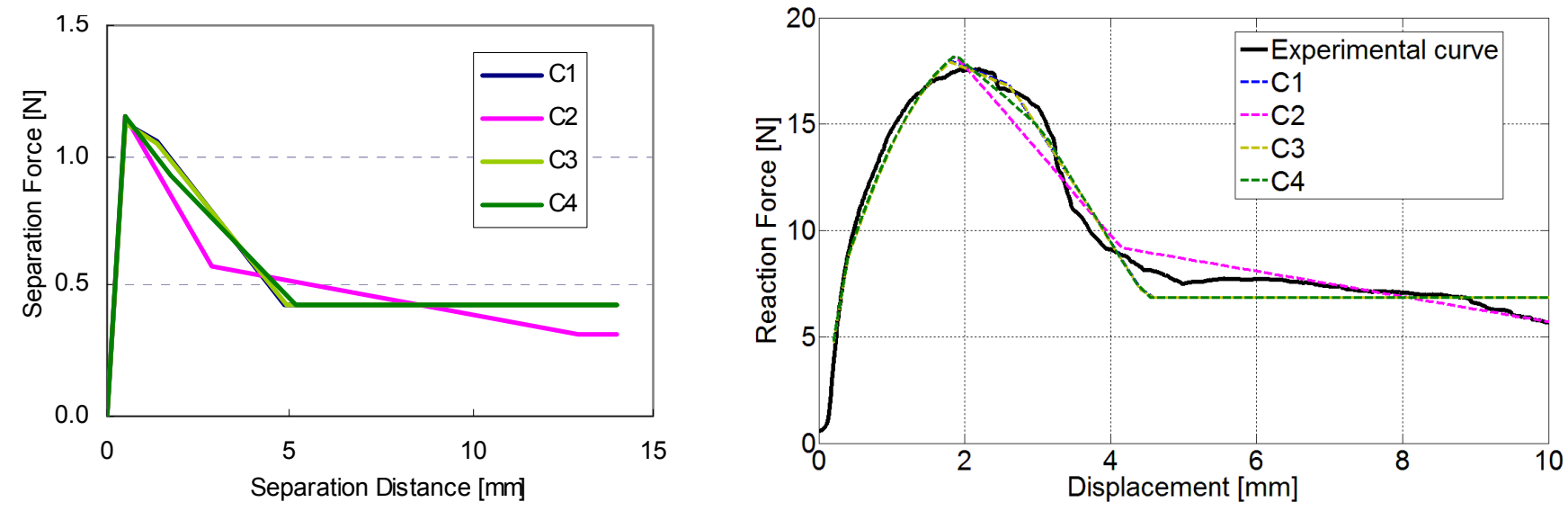

Figure 11: Graphical representation of the identified traction - separation law in the four cases shown in Tab. I (left) and the reconstructed load - displacement curves compared with the experimental output (right); output C2 corresponds to the best matching solution (see Tab. 1).

\begin{tabular}{l|cccccc}
\hline & $\mathbf{F}_{\max }[\mathrm{N}]$ & $\mathbf{F}_{1}[\mathrm{~N}]$ & $\mathbf{F}_{\text {plat }}[\mathrm{N}]$ & $\mathbf{D}_{1}[\mathrm{~mm}]$ & $\mathbf{D}_{2}[\mathrm{~mm}]$ & $\boldsymbol{\omega}_{\text {opt }}$ \\
\hline Initialization & 0.6 & 0.54 & 0.2 & 1.1 & 5.5 & \\
Converged & $\mathbf{1 . 1 5 1}$ & $\mathbf{1 . 0 4 3}$ & $\mathbf{0 . 2 5 0}$ & $\mathbf{1 . 4 4 5}$ & $\mathbf{7 . 0 2 3}$ & $\mathbf{2 2 . 3 5}$ \\
\hline
\end{tabular}

Table 2: Initialization vector and parameter values returned by the minimization of the discrepancy function $\omega$ based on the crack opening profile only.

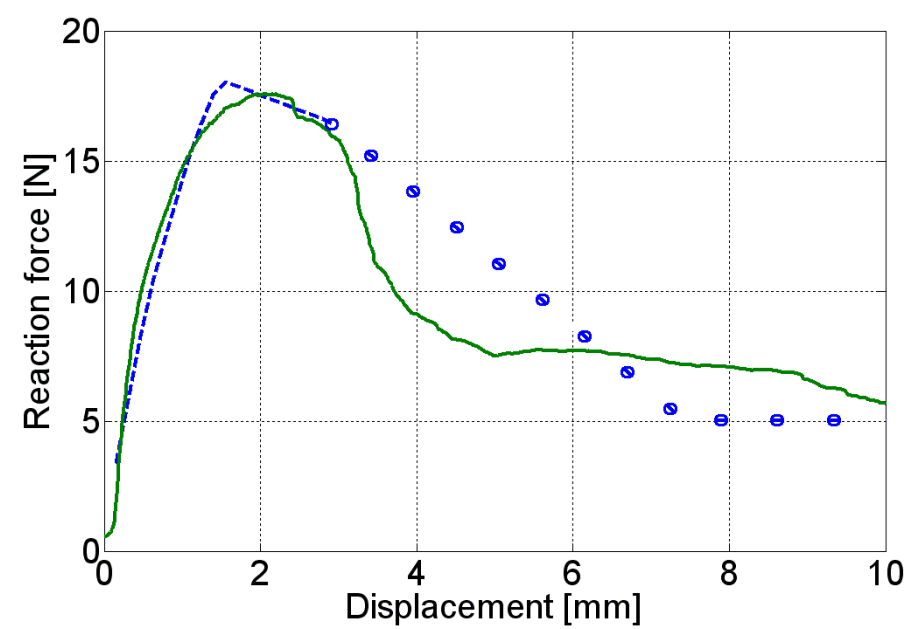

Figure 12: Graphical representation of the experimental output compared with the load - displacement curve reconstructed by the parameter values listed in Tab. 2.

\section{CLOSING REMARKS AND FUTURE PROSPECTS}

$\checkmark$ he combination of properly designed experiments and of the numerical simulation of even complex laboratory tests constitutes the basis of inverse analysis procedures, which are widely exploited to material characterization purposes also in fracture mechanics context. In the presented application, a large amount of information has been collected by means of digital image correlation techniques during non-conventional experiments carried out on heterogeneous material samples. Information concerning crack opening profiles has been extracted and used to calibrate the parameters entering a suitably defined interface model, demonstrating the significant informative content of these deformation data. 


\section{ACKNOWLEDGEMENTS}

his work has been partly supported by a research grant received from Tetra Pak Packaging Solutions (Modena, Italy), which is gratefully acknowledged. Thanks are also due to our Colleagues at the Stazione Sperimentale Carta, Cartone, Pasta per Carta (Milano, Italy) for hosting the experiments in their labs and letting their equipment available for the present investigation.

\section{REFERENCES}

[1] G. Bolzon, M. Talassi, E. Zappa, V. Buljak, In: Atti del XXI Convegno Nazionale IGF, Cassino, Italy (2011).

[2] M.A. Sutton, W.J.Wolters, W.H. Peters, W.F. Ranson, S.R. McNeill, Image and Vision Computing, 1 (1983) 133.

[3] Y.H. Wang, J.H. Jiang, C. Wanintrudal, C. Du, D. Zhou, L.M. Smith, L.X. Yang, Exp. Tech., 34 (2010) 54.

[4] O. Kwon, R. Hanna, Strain, 46 (2010) 566.

[5] S. Sadek, G. Iskander, J. Liu, ASCE J. Comp. Civil Eng., 17 (2003) 88.

[6] D. Zhang, X. Zhang, G. Cheng, Exp. Mech., 39 (1999) 62.

[7] A. Bernasconi, F. Cosmi, E. Zappa, Strain, 46 (2010) 435.

[8] UNI EN ISO 1924-2, Paper and board - Determination of tensile properties, (2009).

[9] D. Lecompte, A. Smits, Sven Bossuyt, H. Sol, J. Vantomme, D. Van Hemelrijck, A.M. Habraken, Optics and Lasers Eng., 44 (2006) 1132.

[10] S. Yaofeng, J.H.L. Pang, Optics and Lasers Eng., 45 (2007) 967.

[11] M.A. Sutton, J.J. Orteu, H.W. Schreier, Image correlation for shape, motion and deformation measurements, Springer Science + Business Media, Heidelberg (2009).

[12] G. Bolzon, R. Fedele, G. Maier, Comput. Meth. Appl. Mech. Engrg., 191 (2002) 2847.

[13] G. Maier, M. Bocciarelli, G. Bolzon, R. Fedele, Int. J. Fract., 138 (2006) 47.

[14] G. Stavroulakis, G. Bolzon, Z. Waszczyszyn, L. Ziemianski, In: Comprehensive Structural Integrity, B. Karihaloo, R.O. Ritchie, I. Milne eds, Elsevier Science Ltd, Kidlington (Oxfordshire), UK, 3 (2003) 685. 